\title{
GENETIC VARIABILITY AND PEDIGREE ANALYSIS OF BRAZILIAN COMMON BEAN ELITE GENOTYPES
}

\author{
Ana Lilia Alzate-Marin ${ }^{1 *}$; Márcia Regina $\mathrm{Costa}^{1}$; Aloísio Sartorato 2 ; María José Del Peloso ${ }^{2}$; \\ Everaldo Gonçalves de Barros ${ }^{1,3}$; Maurilio Alves Moreira ${ }^{1,4}$ \\ ${ }^{1}$ UFV/BIOAGRO, Av. P.H. Rolfs s/n - 36571-000 - Viçosa, MG - Brasil. \\ ${ }^{2}$ Embrapa Arroz e Feijão, C. P. 179 - 75375-000 - Santo Antonio de Goiás, GO - Brasil. \\ ${ }^{3} U F V$ - Depto. de Biologia Geral. \\ ${ }^{4}$ UFV - Depto. de Bioquímica e Biologia Molecular. \\ *Corresponding author <anaalzatem@yahoo.com.br>
}

\begin{abstract}
Genetic diversity is essential for any breeding program. However, breeders tend to concentrate on specific genotypes, which combine traits of interest and may be used as progenitors in several breeding programs. Common bean (Phaseolus vulgaris L.) breeding programs are not different in this sense. In this study, the genetic diversity of 21 common bean elite lines from the Bean Regional Trials conducted by the Embrapa Rice and Bean Research Center was evaluated using the Random Amplified Polymorphic DNA (RAPD) and pedigree analyses. Based on genetic dissimilarity, three groups were defined: group I - lines 1, 9 and 10 , with low genetic distances among them $(0.00$ to 0.06$)$, originated from 11 Mesoamerican parents; group II - 17 lines with genetic distances ranging from 0.03 to 0.33 , originated from 50 parents (mostly Mesoamerican); and group III - line 21 (PR 93201472), which parents are the Andean cultivar 'Pompadour' and the cultivar 'Irai' (unknown origin). The genetic distances between line 21 and the lines of the other two groups varied from 0.68 to 0.93 . Pedigree analyses demonstrated that cultivars 'Carioca', 'Cornell 49-242', 'Jamapa', 'Tlalnepantla 64', 'Tara' and 'Veranic 2', all of Mesoamerican origin, were the most widely used parents for developing lines present in group II.
\end{abstract}

Key words: Phaseolus vulgaris, Andean cultivars, Mesoamerican cultivars, RAPD molecular markers, genetic distances

\section{VARIABILIDADE GENÉTICA E ANÁLISE DE PEDIGREE EM GENÓTIPOS ELITE BRASILEIROS DE FEIJOEIRO COMUM}

\begin{abstract}
RESUMO: Diversidade genética é um pré-requisito em qualquer tipo de programa de melhoramento. No entanto, os melhoristas tendem a se concentrar em alguns genótipos que reúnem características de interesse e estes são usados em diversos programas de melhoramento. Os programas de melhoramento do feijoeiro (Phaseolus vulgaris L.) não são diferentes quanto a esse aspecto. Visando o estudo da variabilidade genética, 21 cultivares-elite dos Ensaios Regionais de Feijão coordenados pela Embrapa Arroz e Feijão, foram caracterizados com marcadores moleculares Random Amplified Polymorphic DNA (RAPD). Também, os pedigrees dos 21 cultivares elite foram pesquisados com o objetivo de estudar os progenitores usados no seu desenvolvimento. Baseado nos dados de distância genética, um gráfico de dispersão foi construído e três grupos foram identificados: 1) grupo I, formado pelas linhas 1,9 e 10, com baixa diversidade genética entre si $(0,00$ a 0,06$)$, originadas de 11 progenitores de origem Mesoamericana; 2$)$ grupo II, formado por 17 linhagens com distâncias genéticas variando de 0,03 a 0,33 , originadas de 50 progenitores, a maioria de origem Mesoamericana; e, 3) grupo III, formado pelo cultivar PR 93201472 (linhagem 21), que tem os cultivares 'Pompadour' (origem Andina) e 'Irai' (origem desconhecida) como seus progenitores. As distâncias genéticas entre PR 93201472 e os outros 20 cultivares variaram entre 0,68 e 0,93 . De acordo com seus pedigrees, os cultivares 'Carioca', 'Cornell 49-242', 'Jamapa', 'Tlalnepantla 64', 'Tara' e 'Veranic 2', todos de origem Mesoamericana, foram os progenitores mais empregados na geração das linhagens do grupo II.

Palavras-chave: Phaseolus vulgaris L., cultivares Andinos, cultivares Mesoamericanos, marcadores moleculares RAPD, distâncias genéticas
\end{abstract}

\section{INTRODUCTION}

Brazil, Mexico, Argentina and United States are the leading common bean (Phaseolus vulgaris) producers in the world. In Latin America and Africa, beans are major staple food and an important protein source. Brazil is the world leading producer of Phaseolus beans, but its production is destined to internal consumption. The U.S. is the leading exporter marketing $40 \%$ ( 0.4 million metric tons) of all their dry bean production overseas, 
while Argentina exports $99.5 \%$ of its production of 0.25 million metric tons (Phaseolus Crop Germplasm Committee Report, 1996; FAO, 1998).

Studies on variation patterns of the seed protein phaseolin, alloenzymes and morphological evidences, revealed the existence of a Middle American and an Andean gene pools in Phaseolus vulgaris (Gepts et al., 1986; Singh et al., 1991a; Singh et al., 1991b). Phaseolin $\mathrm{S}$ is common among the three Phaseolus races in the Middle American gene pool, while $\mathrm{Sb}, \mathrm{Sd}$ and $\mathrm{B}$ phaseolins are less common. On the other hand, phaseolin $\mathrm{T}, \mathrm{C}$ and $\mathrm{H}$ are present in genotypes from the Andean gene pool. Not only the phaseolin patterns but also the size of the seeds differentiates Middle American from Andean gene pools. Mesoamerican landraces have usually smaller seeds than their Andean counterparts (Gepts, et al., 1986; Singh et al., 1990).

Biochemical studies have shown that the cultivated bean germplasm presents a low level of genetic diversity as compared to their wild ancestors (Gepts et al., 1986; Koenig et al., 1990; Sonnante et al., 1994; Johnson \& Gepts, 1998). Progress in bean breeding around the world has been slow, probably because of a limited genetic variability of the parents which have been selected from the same gene pool.

Isozymes and DNA-based markers have been used to evaluate germplasm genetic diversity in Phaseolus species, within both cultivated and wild genetic materials; to analyze crop evolution; and the effect of domestication on genetic diversity (Gepts, 1988; Singh et al., 1990; Koening et al., 1990; Chase et al., 1991; Singh et al., 1991a, b, c; Arraes et al., 1992; Thome et al., 1996; Nienhuis et al., 1996; Alvarez et al., 1998, Vasconcelos, 1995; Beebe et al., 2000). Métais et al. (2000) evaluated the effectiveness of Restriction Fragment Length Polymorphism (RFLP), Direct Amplified Minisatellite DNA-PCR (DAMD-PCR), Inter Simple Sequence Repeat (ISSR) and Random Amplified Polymorphic DNA (RAPD) markers in the assessment of polymorphism and relationships between commercial lines of $P$. vulgaris. RAPD and RFLP analyses led to the same clustering of the bean lines. All analyzed genotypes could be distinguished by only seven RAPD markers.

The objectives of the present study were: to evaluate the genetic variability within 21 elite cultivars from the Bean Regional Trials coordinated by Embrapa Rice and Bean Research Center using RAPD markers; and to identify the parents involved in the development of these cultivars through pedigree analyses.

\section{MATERIAL AND METHODS}

The pedigree of each of the 21 elite lines from Regional Trails used in this study is shown in Table 1.
Primary leaves from each individual line were collected, identified and stored at $-80^{\circ} \mathrm{C}$. DNA extraction was made according to Doyle \& Doyle (1990). Amplification reactions of $25 \mu \mathrm{L}$ each, contained $25 \mathrm{ng}$ of DNA, $0.1 \mathrm{mmol}$ $\mathrm{L}^{-1}$ of each dNTP (dATP, dCTP, dGTP, dTTP), $2.0 \mathrm{mmol}$ $\mathrm{L}^{-1}$ of $\mathrm{MgCl}_{2}, 10 \mathrm{mmol} \mathrm{L}^{-1}$ Tris- $\mathrm{HCl}, \mathrm{pH} 8.3,50 \mathrm{mmol} \mathrm{L}^{-1}$ $\mathrm{KCl}, 0.4 \mu \mathrm{mol} \mathrm{L}^{-1}$ of one primer decamer (Operon Technologies, Alameda, CA, USA), and one unit of Taq DNA polymerase (Williams et al., 1990).

Each amplification cycle consisted of one denaturation step at $94^{\circ} \mathrm{C}$ for $15 \mathrm{~s}$, one annealing step at $35^{\circ} \mathrm{C}$ for $30 \mathrm{~s}$, and one extension step at $72^{\circ} \mathrm{C}$ for $1 \mathrm{~min}$. After 40 cycles, an extra extension step was performed for $7 \mathrm{~min}$ at $72^{\circ} \mathrm{C}$. Amplification products were analyzed on $1.2 \%$ agarose gels immersed on TBE buffer $\left(90 \mu \mathrm{mol} \mathrm{L}^{-1}\right.$ Trisborate; $2 \mu \mathrm{mol} \mathrm{L}{ }^{-1}$ EDTA, pH 8.0) containing $10 \mu \mathrm{g}$ of ethidium bromide per mL (Williams et al., 1990). DNA bands were visualized under UV light and photographed with the Eagle Eye II photosystem (Stratagene, La Jolla, CA, USA).

Fourteen primers were tested and only two did not show polymorphic bands. The primers OPB 15 , OPB18, OPAT05, OPY10, OPAA18, OPBA18, OPBB20, OPAW19, OPAX03, OPAZ18, OPAX02 and OPAX18 revealed polymorphism. Only the strong and reproducible bands were used for analyses.

The clustering analysis was based on the genetic distance calculated by the Nei \& Li method for binary data, using the GENES computer program (Cruz, 1997). Based on the genetic distances a dispersion graphic on two-dimensional space was obtained (Cruz \& Soriano, 1994).

\section{RESULTS AND DISCUSSION}

General information on cultivars/lines involved in the breeding program to obtain the elite lines of the Bean Regional Trials is presented in Table 2. The DNA amplification results presented 32 polymorphic and 46 monomorphic bands, among the 21 bean cultivars. Based on the genetic distances a two-dimensional dispersion graph was obtained. In the dispersion graph, it was possible to distinguish three different groups (Table 3, Figure 1).

The first group was formed by three black seed lines with identical genealogies, LR 9115398 (1), AN 9021334 (9) and AN 9021336 (10), derived from 11 Mesoamerican parents. These lines presented low genetic distances between them ( 0.00 to 0.06$)$ (Figure 1). Lines (9) and (10) showed a genetic distance of zero, while line (1) presented a genetic distance of 0.06 in relation to lines (9) and (10) (Table 3). These lines carries anthracnose and rust resistance genes, derived from the cultivar Honduras 35 known in Brazil as Ouro Negro (Faleiro et al., 2000; Alzate-Marin et al., 2001). 
Table 1 - Seed color and pedigrees of the 21 elite common bean lines of the Bean Regional Trials coordinated by Embrapa Rice and Bean.

\begin{tabular}{|c|c|c|c|}
\hline BRT line ${ }^{a}$ & Color & Size $^{\mathrm{d}}$ & Pedigree \\
\hline 1. LR 9115398 & Black $^{\mathrm{b}}$ & Small (20.5) & $\begin{array}{l}(((\text { G } 2698 \times(\text { BRASIL } 343 \times \text { BRASIL 1096) }) \times(\text { CAUCA } 41)) \times(\text { ICA TUI x S } 166 \text { AN }) \times \\
(\text { G } 2084 \times 51051) \times(\text { SB 12))x (XAN 87)) }) / \text { HONDURAS } 35\end{array}$ \\
\hline 2. LR 9115453 & Brown & Small (20.4) & $\begin{array}{l}(\text { DRO } 4784) /((\text { VERANIC } 2 \times \text { TLALNEPANTLA } 64) \times(\text { JAMAPA } \times \text { TARA }) \times \\
\text { PORRILLO SINTETICO x PI } 310878) \times \text { NEP BAYO 22))// (AROANA x ((VERANIC } \\
2 \times \text { TLALNEPANTLA 64) x ( JAMAPA x TARA) ) / (OJO DE LIEBRE x ((PORRILLO } \\
\text { SINTETICO x CACAHUATE 73) x (JAMAPA x CACAHUATE } 72)))\end{array}$ \\
\hline 3. A 774 & Brown & Small (20.4) & $\begin{array}{l}((51051 \times I C A \text { BUNSI)x }(51052 \times \text { CORNELL } 49242)) / / /(\text { CARIOCA x MEX. 168)/ } \\
\text { CARIOCA } 80 \text { // (CARIOCA x MEX. 168)/((ICA TUI x TLALNEPANTLA 64) x } \\
\text { (PORRILLO SINTETICO x JULES)) }\end{array}$ \\
\hline 4. PR 9115957 & Brown & Small (22.0) & (TLALNEPANTLA $64 \times$ AROANA) / GOIANO PRECOCE \\
\hline 5. FEB 163 & Purple & Small (16.8) & 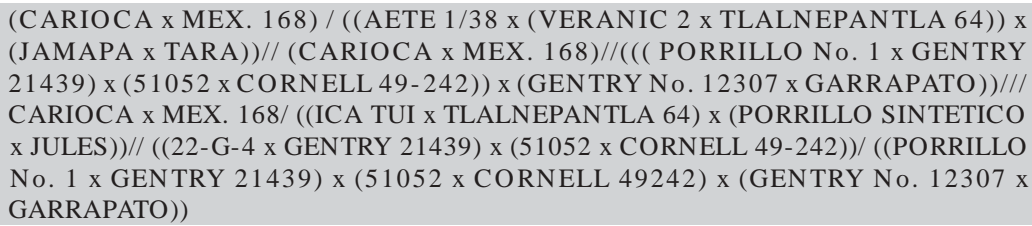 \\
\hline 6. RAO 33 & Red & Small (24.4) & $\begin{array}{l}(((\text { POMPADOUR CHECA } \times(\mathrm{G} 03645 \times \mathrm{G} 02045)) \times(\mathrm{G} 03974 \times \mathrm{G} 04485)) /(51052 \times \\
\text { COPAN })=((\text { POMPADOUR CHECA } \times(\text { JAMAPA } \times \text { GENTRY } 21439)) \times(\mathrm{JIN} 10 \times \\
\text { TURRIALBA } 1)) /(51052 \times \text { COPAN })\end{array}$ \\
\hline 7. LM 93204217 & Black $^{c}$ & Small (21.2) & $\begin{array}{l}((\text { PORRILLO SINTÉTICO } \times \text { TURRIALBA } 1) \times(\text { ICA PIJAO } \times \text { NEGRO JAMAPA })) / / \\
(((\text { VERANIC } 2 \times \text { TLALNEPANTLA 64)F } 1 \times((\text { JAMAPA } \times \text { TARA }) \text { F } 1 \times \text { AETE } 1 / 37))) / \\
(((\text { IPA } 7419 \times(\text { HONDURAS } 46 \times \text { VENEZUELA 54))///((AROANA } \times((\text { VERANIC } 2 \times \\
\text { TLALNEPANTLA 64) x ( JAMAPA x TARA }))) / /(\text { G } 4326 /((\text { S } 166 \text { AN x ECUADOR } \\
\text { 299) x ((VERANIC } 2 \times \text { TLALNEPANTLA 64) X (JAMAPA X TARA)))) }\end{array}$ \\
\hline 8. TB 94-01 & Black & Small (18.4) & 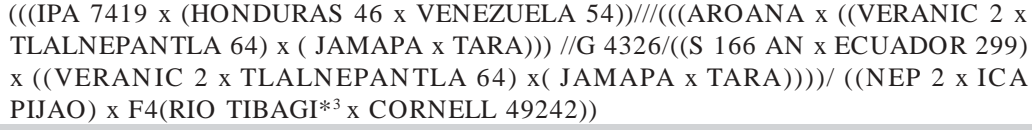 \\
\hline 9. AN 9021334 & Black & Small (18.8) & SAME AS LR 9115398 \\
\hline 10. AN 9021336 & Black & Small (17.8) & SAME AS LR 9115398 \\
\hline 11. LM 93204303 & Cream-beige & Small (13.9) & $\begin{array}{l}\text { (CARIOCA/RIO TIBAGI) / (((CARIOCA x }((\text { PI } 307824 \text { x PI } 310797) \times(\text { TURRIALBA } \\
4 \text { x CORNELL 49-242))/// (CARIOCA x MEX. 168)//(( AETÉ 1/38 x ((VERANIC } 2 \times \\
\text { TLALNEPANTLA 64) x (JAMAPA x TARA)))/ ((VERANIC } 2 \text { x TLALNEPANTLA 64) } \\
\text { x ( JAMAPA x TARA) x AETÉ 1/37))) }\end{array}$ \\
\hline 12. LM 93204319 & Cream-beige & Small (17.0) & (CARIOCA/RIO TIBAGI) / ((51052 x CACAHUATE 72) x CARIOCA*2) \\
\hline 13. LM 93204328 & Cream-beige & Small (17.8) & SAME AS LM 93204319 \\
\hline 14. LM 93204453 & Cream-beige & Small (19.4) & 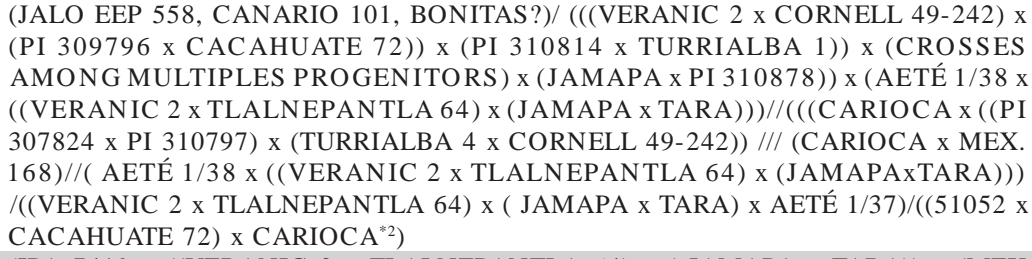 \\
\hline 15. AN 9021470 & Brown & Small (18.8) & $\begin{array}{l}(\text { IPA-7419 } \times((\text { VERANIC } 2 \times \text { TLALNEPANTLA } 64) \times(\text { JAMAPA } \times \text { TARA })) \times(\text { MEX } \\
168)) /((\text { IPA } 7419 \times(\text { HONDURAS } 46 \times \text { VENEZUELA 54) }) / / /(\text { AROANA } \times((\text { VERANIC } \\
2 \times \text { TLALNEPANTLA 64) } \times(\text { JAMAPA } \times \text { TARA })) / / G 4326 /((\text { S } 166 \text { AN } \times \text { ECUADOR } \\
299) \times((\text { VERANIC } 2 \times \text { TLALNEPANTLA 64) }(\text { JAMAPA } \times \text { TARA })))\end{array}$ \\
\hline 16. LM 9220225 & Brown & Small (18.9) & $\begin{array}{l}\text { DRO } 4784 \text { / }((((\text { VERANIC } 2 \text { x CORNELL } 49-242) \text { x ( PI } 309796 \text { x CACAHUATE } 72)) \\
\text { x (PI } 310814 \text { x TURRIALBA 1)) x (CROSSES AMONG MULTIPLES PROGENITORS) } \\
\text { x (JAMAPA x PI 310878))) x (AETÉ } 1 / 38 \text { x ((VERANIC } 2 \text { x TLALNEPANTLA 64) x } \\
(\text { (JAMAPA x TARA })))\end{array}$ \\
\hline 17. L 96029 & Brown & Small (13.1) & $\begin{array}{l}(((\text { VERANIC } 2 \times \text { TLALNEPANTLA 64)F } 1 \text { x (JAMAPA x TARA)F } 1) \times \text { AETE 1/37) / } \\
\text { (LINEA } 32 \text { x TURRIALBA 1) }\end{array}$ \\
\hline 18. LM 93203246 & Pink & Small (16.3) & ADVANCED MATERIAL RECEIBED FROM CIAT 1981/ ROSINHA G2 RMC \\
\hline 19. LM 93203304 & Pink & Small (23.6) & $\begin{array}{l}\text { HI822510?/ (((ICA } 10310 \times((\text { VERANIC } 2 \text { x TLALNEPANTLA 64) } \times \text { (TURRIALBA } 4 \\
\text { x CORNELL 49-242)) x (( S } 166 \text { AN } 5 \text { 51054) x ((VERANIC } 2 \text { x TLALNEPANTLA } \\
\text { 64) x (JAMAPA x TARA))) // (ADVANCED MATERIAL RECEIBED FROM CIAT 1981/ } \\
\text { ROSINHA G2 RM) }\end{array}$ \\
\hline 20. LR 93201684 & Purple & Small (21.5) & $\begin{array}{l}\text { CF (BEAN HARVEST) / ((POMPADOUR CHECA x ((JAMAPA x GENTRY 21439) } \mathrm{x} \\
(\text { JIN } 10 \times \text { TURRIALBA } 1))) \times(51052 \times \text { COPAN }))\end{array}$ \\
\hline 21. PR 93201472 & Manteigão & Medium (40.0) & POMPADOUR/IRAI \\
\hline
\end{tabular}


Table 2 - General information on cultivars/lines involved in the breeding program to obtain the elite lines of the Bean Regional Trials coordinated by Embrapa Rice and Beans.

\begin{tabular}{|c|c|c|c|c|}
\hline & CULTIVAR/ LINE & $\begin{array}{l}\text { ELITE } \\
\text { LINES - TABLE } 1\left({ }^{\mathrm{n}}=\text { total }\right. \\
\text { usage frequency of these } \\
\text { cultivars/lines })\end{array}$ & $\begin{array}{l}\text { CULTIVAR/LINE } \\
\text { ORIGIN }\end{array}$ & NOTE \\
\hline 1 & 51051 & $1^{1}, 3^{1}, 9^{1}, 10^{1}$ & Costa Rica & Rust differential ${ }^{\mathrm{a}}$ \\
\hline 2 & 51052 & $3^{1}, 5^{3}, 6^{1}, 12^{1}, 14^{1}, 20^{1}$ & Costa Rica & Turrialba $4 \mathrm{~N}^{\text {bd }}$ \\
\hline 3 & 51054 & $19^{1}$ & Costa Rica & $\mathrm{b}$ \\
\hline 4 & AETÉ $1 / 37$ & $11^{1}, 14^{1}$ & Brazil & Parana - Brazil ${ }^{\mathrm{b}}$ \\
\hline 5 & AETE $1 / 38$ & $11^{1}, 14^{2}, 16^{1}$ & Brazil & b \\
\hline 6 & AROANA & $2^{1}, 4^{1}, 7^{1}, 8^{1}, 15^{1}$ & Brazil & $\begin{array}{l}\text { Chumbinho } 79 \times \text { Actopan (Mexico) (brown and } \\
\text { small seed) })^{\mathrm{d}}\end{array}$ \\
\hline 7 & BAT 477 & $3^{1}$ & Colombia (CIAT) & (51051 x ICA Bunsi) x (51052 x Cornell 49-242) \\
\hline 8 & BRASIL 1096 & $1^{1}, 9^{1}, 10^{1}$ & Brazil & \\
\hline 9 & BRASIL 343 & $1^{1}, 9^{1}, 10^{1}$ & Brazil & Brown seeds \\
\hline 10 & CACAHUATE 72 & $2^{1}, 12^{1}, 13^{1}, 14^{2}, 16^{1}$ & Mexico (list of CIAT) & $\begin{array}{l}\text { Seed yield high. Moderate resistance to bean rust } \\
\text { and angular leaf spot. Resistant to bean common } \\
\text { mosaic virus (single dominant I-gene). Inbred } \\
\text { backcross (BC253) line from ICA Pijao/Puebla } 152^{\mathrm{c}}\end{array}$ \\
\hline 11 & CACAHUATE 73 & $2^{1}$ & Mexico & $\begin{array}{l}\text { Seed yield high. Moderate resistance to bean rust } \\
\text { and angular leaf spot. Resistant to bean common } \\
\text { mosaic virus (single dominant I-gene). Inbred } \\
\text { backcross (BC253) line from ICA Pijao/Puebla } 152^{\mathrm{c}}\end{array}$ \\
\hline 12 & CARIOCA & $3^{2}, 5^{3}, 11^{3}, 12^{2}, 13^{2}, 14^{3}$ & 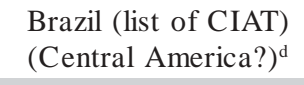 & $\begin{array}{l}\text { Resistant to Macrophomina phaseolina (Mp) } \\
\text { (Mayek-Perez et al., 1999)/ Race Middle American }^{\mathrm{c}}\end{array}$ \\
\hline 13 & CARIOCA 80 & $3^{1}$ & Brazil (list of CIAT) & \\
\hline 14 & CAUCA 41 & $1^{1}, 9^{1}, 10^{1}$ & Colombia & Negrito Chiquito - G $2515^{\mathrm{bc}}$ \\
\hline 15 & COPAN & $6^{1}, 20^{1}$ & Honduras & Mexico $80 \times$ BAT $724^{\mathrm{d}}$ \\
\hline 16 & $\begin{array}{l}\text { CORNELL 49- } \\
242\end{array}$ & $3^{1}, 5^{3}, 8^{1}, 11^{1}, 14^{2}, 16^{1}$ & $\begin{array}{c}\text { Venezuela } \\
(\text { Central America? })^{\mathrm{d}}\end{array}$ & $\begin{array}{l}I \text { and } b c-u \text { genes. Middle American (Vasconcelos, } \\
\text { 1995). Anthracnose differential. }\end{array}$ \\
\hline 17 & DRO 4784 & $2^{1}, 16^{1}$ & Colombia (CIAT) & \\
\hline 18 & ECUADOR 299 & $7^{1}, 8^{1}, 15^{1}$ & Ecuador & $\begin{array}{l}\text { Rust differential. Gene } U r-3 \text {. Resistant to Andean } \\
\text { isolates }^{\mathrm{a}}\end{array}$ \\
\hline 19 & G 4326 & $7^{1}, 8^{1}, 15^{1}$ & Mexico & Zacatecas (list of CIAT) \\
\hline 20 & G 2084 & $1^{1}, 9^{1}, 10^{1}$ & Nicaragua & Gentry 21555 (list of CIAT) \\
\hline 21 & G 2698 & $1^{1}, 9^{1}, 10^{1}$ & Venezuela & S-234 (Venezuela 23) (list of CIAT) \\
\hline 22 & GARRAPATO & $5^{2}$ & Mexico (list of CIAT) & BGMV resistance source (Urrea et al., 1996) \\
\hline 23 & GENTRY 21439 & $6^{1}, 20^{1}$ & Guatemala & (list of CIAT) \\
\hline 24 & GENTRY 12307 & $5^{2}$ & Mexico & PI203937. G 879 \\
\hline 25 & $\begin{array}{l}\text { GOIANO } \\
\text { PRECOCE }\end{array}$ & $4^{1}$ & Brazil & \\
\hline 26 & HONDURAS 35 & $1^{1}, 9^{1}, 10^{1}$ & Honduras $^{\mathrm{d}}$ & $\begin{array}{l}\text { Middle American (Vasconcelos, 1995). Cultivar } \\
\text { Ouro Negro. }\end{array}$ \\
\hline 27 & HONDURAS 46 & $7^{1}, 8^{1}, 15^{1}$ & Honduras & Small dark and brilliant seeds ${ }^{d}$ \\
\hline
\end{tabular}


Table 2 - Continuation.

\begin{tabular}{|c|c|c|c|c|}
\hline & CULTIVAR/ LINE & $\begin{array}{c}\text { ELITE } \\
\text { LINES - TABLE } 1\left(^{\mathrm{n}}=\text { total }\right. \\
\text { usage frequency of these } \\
\text { cultivars/lines })\end{array}$ & $\begin{array}{l}\text { CULTIVAR/LINE } \\
\text { ORIGIN }\end{array}$ & NOTE \\
\hline 28 & ICA BUNSI & $3^{1}$ & Colombia & Magdalena $8 \times$ Japon $3 \frac{\mathrm{d}}{I}$ and $b c-u$ genes \\
\hline 29 & ICA PIJAO & $7^{1}, 8^{1}$ & Colombia & Porrillo Sintético x Mexico 11 \\
\hline 30 & ICA TUI & $1^{1}, 3^{1}, 5^{1}, 9^{1}, 10^{1}$ & Colombia $^{\mathrm{d}}$ & Selection in Venezuela $44^{\mathrm{d}} / I$ and $b c-u$ genes $^{\mathrm{a}}$ \\
\hline 31 & IRAI & $21^{1}$ & Brazil & \\
\hline 32 & IPA 7419 & $7^{1}, 8^{1}, 15^{2}$ & Brazil & L 3-0-50 x Costa Rica \\
\hline 33 & JAMAPA & $\begin{array}{l}2^{3}, 5^{3}, 6^{1}, 7^{3}, 8^{2}, 11^{2}, 14^{4} \\
\quad 15^{3}, 16^{2}, 17^{1}, 19^{1}, 20^{1}\end{array}$ & Mexico $^{d}$ & $\begin{array}{l}\text { Mixture of } 15 \text { lines from Veracruz } 87 \text { collection/I and } b c \text { - } \\
u \text { genes / Middle American (Singh et al., 1991a). } \\
\text { Resistant to Macrophomina phaseolina (Mayek-Perez } \\
\text { et al., 1999)/ Mesoamerica race (Singh et al., 1991a) }\end{array}$ \\
\hline 34 & JIN 10 & $6^{1}, 20^{1}$ & Nicaragua & (list of CIAT) \\
\hline 35 & JULES & $3^{1}, 5^{1}$ & $\begin{array}{l}\text { United States of } \\
\text { America }\end{array}$ & GN Nebraska \#1 sel. $27 /$ GN 1140 (= to Tara) ${ }^{b}$ \\
\hline 36 & LINEA 32 & $17^{1}$ & $\begin{array}{l}\text { Colombia (List of } \\
\text { CIAT) }\end{array}$ & $\begin{array}{l}\text { (PI } 203958 \text { x Red Mexican UI-35) x a curly top- } \\
\text { resistant early short bush Dark Red Kidney type } \\
\text { breeding line, DRK } 801 \times \text { Sutter Pink. Mexican bean } \\
\text { with effective level field resistant to fusarium root } \text { rot }^{c}\end{array}$ \\
\hline 37 & MEX. 168 & $3^{2}, 5^{3}, 11^{1}, 14^{1}, 15^{1}$ & Mexico & $\begin{array}{l}\text { Rio Tibagi x Guanajuato } 31 \text { (Mesoamerica x Durango } \\
\text { races) }{ }^{c} \text {, Anthracnose resistance source }\end{array}$ \\
\hline 38 & NEGRO JAMAPA & $7^{1}$ & Mexico & Gentry 21157 (list of CIAT) \\
\hline 39 & NEP 2 & $8^{1}$ & Costa Rica & $\begin{array}{l}\text { Rust differential resistant to Andean isolates }{ }^{\mathrm{a}} \text {. Small white } \\
\text { seed from cultivar San Fernando }(\mathrm{S} 182 \mathrm{~N}) \text {, selection } \\
\text { from Turrialba through mutagenesis }{ }^{\mathrm{d}} / I \text { and } b c-u \text { genes } \\
\text { (Middle American) }\end{array}$ \\
\hline 40 & NEP BAYO 22 & $2^{1}$ & Costa Rica & d \\
\hline 41 & OJO DE LIEBRE & $2^{1}$ & Mexico & '(Gentry 22129, Gentry 22538) \\
\hline 42 & PI 307824 & $11^{1}, 14^{1}$ & El Salvador & Porrillo 1c \\
\hline 43 & PI 309796 & $14^{1}, 16^{1}$ & Mexico & Jalisco (Mex). Gentry $21149^{\mathrm{bc}}$ \\
\hline 44 & PI 310797 & $11^{1}, 14^{1}$ & Guatemala & Huehuetenango $(\mathrm{G})^{\mathrm{bc}}$ \\
\hline 45 & PI 310814 & $14^{1}, 16^{1}$ & Nicaragua & Gentry 21556. Granada $(\mathrm{N})^{\mathrm{c}}$ \\
\hline 46 & PI 310878 & $2^{1}, 14^{1}, 16^{1}$ & Nicaragua & Rojo Claro ${ }^{c}$ \\
\hline 47 & $\begin{array}{l}\text { POMPADOUR } \\
\text { (CHECA) }\end{array}$ & $6^{1}, 20^{1}$ & Dominican Republic & $\begin{array}{l}\text { Andean, rust resistant (Bassett, 1996). Red and pink } \\
\text { mottled median size seeds Selected from cultivar } \\
\text { Pompadour }\end{array}$ \\
\hline 48 & POMPADOUR & $21^{1}$ & Dominican Republic & d \\
\hline 49 & PORRILLO No. 1 & $5^{2}$ & El Salvador & $\begin{array}{l}\text { Selected from cultivar Santa Clara (Venezuela)d/ Middle } \\
\text { American }\end{array}$ \\
\hline 50 & $\begin{array}{l}\text { PORRILLO } \\
\text { SINTETICO }\end{array}$ & $2^{2}, 3^{1}, 5^{1}$ & El Salvador & 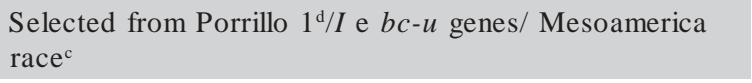 \\
\hline 51 & RIO TIBAGI & $8^{1}, 11^{1}, 12^{1}, 13^{1}$ & Costa Rica & Selected from cultivar S $89 \mathrm{~N}^{\mathrm{d}} / \mathrm{Mesoamerica}$ race $^{\mathrm{c}}$ \\
\hline 52 & $\begin{array}{l}\text { ROSINHA } \\
\text { G2RMC }\end{array}$ & $18^{1}, 19^{1}$ & Brazil & \\
\hline 53 & S 166 AN & $1^{1}, 7^{1}, 8^{1}, 9^{1}, 10^{1}, 15^{1}, 19^{1}$ & Costa Rica & d \\
\hline 54 & SB 12 & $1^{1}, 9^{1}, 10^{1}$ & Zambia & Gandajika A 65. Arachis hypogaea \\
\hline 55 & TARA & $\begin{array}{c}2^{2}, 5^{1}, 7^{2}, 8^{1}, 11^{2}, 14^{3}, 15^{3} \\
16^{1}, 17^{1}, 19^{1}\end{array}$ & $\begin{array}{l}\text { United States of } \\
\text { America }\end{array}$ & $\begin{array}{l}\text { GN Nebraska \#1 Sel. } 27 / \mathrm{GN} 1140 \text { (= to Jules) }^{\mathrm{b}} \text {. } \\
\text { Resistant to some pathotypes of } U \text {. appendiculatus. } \\
\text { Tolerant to the bean common bacterial blight. Developed } \\
\text { in Nebraskac. }\end{array}$ \\
\hline 56 & $\begin{array}{l}\text { TLALNEPANTLA } \\
64\end{array}$ & $\begin{array}{l}2^{2}, 3^{1}, 4^{1} 5^{2}, 7^{3}, 8^{2}, 11^{2} \\
14^{3}, 15^{3}, 16^{1}, 17^{1}, 19^{2}\end{array}$ & Mexico & Collected in 1943.PI 207.262, Mexico 56, G $1320^{\text {bc }}$ \\
\hline 57 & TURRIALBA 1 & $6^{1}, 7^{1}, 14^{1}, 16^{1}, 17^{1}, 19^{1}$ & Costa Rica & $\begin{array}{l}\text { S } 19 \mathrm{~N} \text { - Centro American bulk selection } / / I \text { and } b c-u \\
\text { genes }\end{array}$ \\
\hline 58 & TURRIALBA 4 & $11^{1}, 14^{1}, 19^{1}$ & Costa Rica & Individual selection from Guatemala ${ }^{\mathrm{d}} / I$ and $b c-u$ genes. \\
\hline 59 & VENEZUELA 54 & $7^{1}, 8^{1}, 15^{1}$ & Venezuela & c \\
\hline 60 & VERANIC 2 & $\begin{array}{c}2^{2}, 5^{1}, 7^{3}, 8^{2}, 11^{2}, 14^{4}, 15^{3} \\
16^{2}, 17^{1}, 19^{2}\end{array}$ & Nicaragua & Selected from Jamapa (Mexico) ${ }^{d}$ \\
\hline 61 & XAN 87 & $1^{1}, 9^{1}, 10^{1}$ & Colombia (CIAT) & $\left(\right.$ BAT $450 \times$ SEL 29) ${ }^{d}$ \\
\hline
\end{tabular}

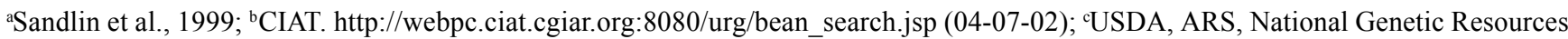
Program. Germplasm Resources Information Network - (GRIN). [Online Database] National Germplasm Resources Laboratory, Beltsville, Maryland. Available: www.ars-grin.gov/cgi-bin/npgs/html/acc_search.pl (02-07-02); ${ }^{\mathrm{d}}$ Voysest, 1983; ${ }^{\mathrm{e}}$ Balardin \& Pastor Corrales, 1990. 
Table 3 - Genetic distances among pairs of common bean elite cultivars from the Regional Trials coordinated by Embrapa Rice and Beans.

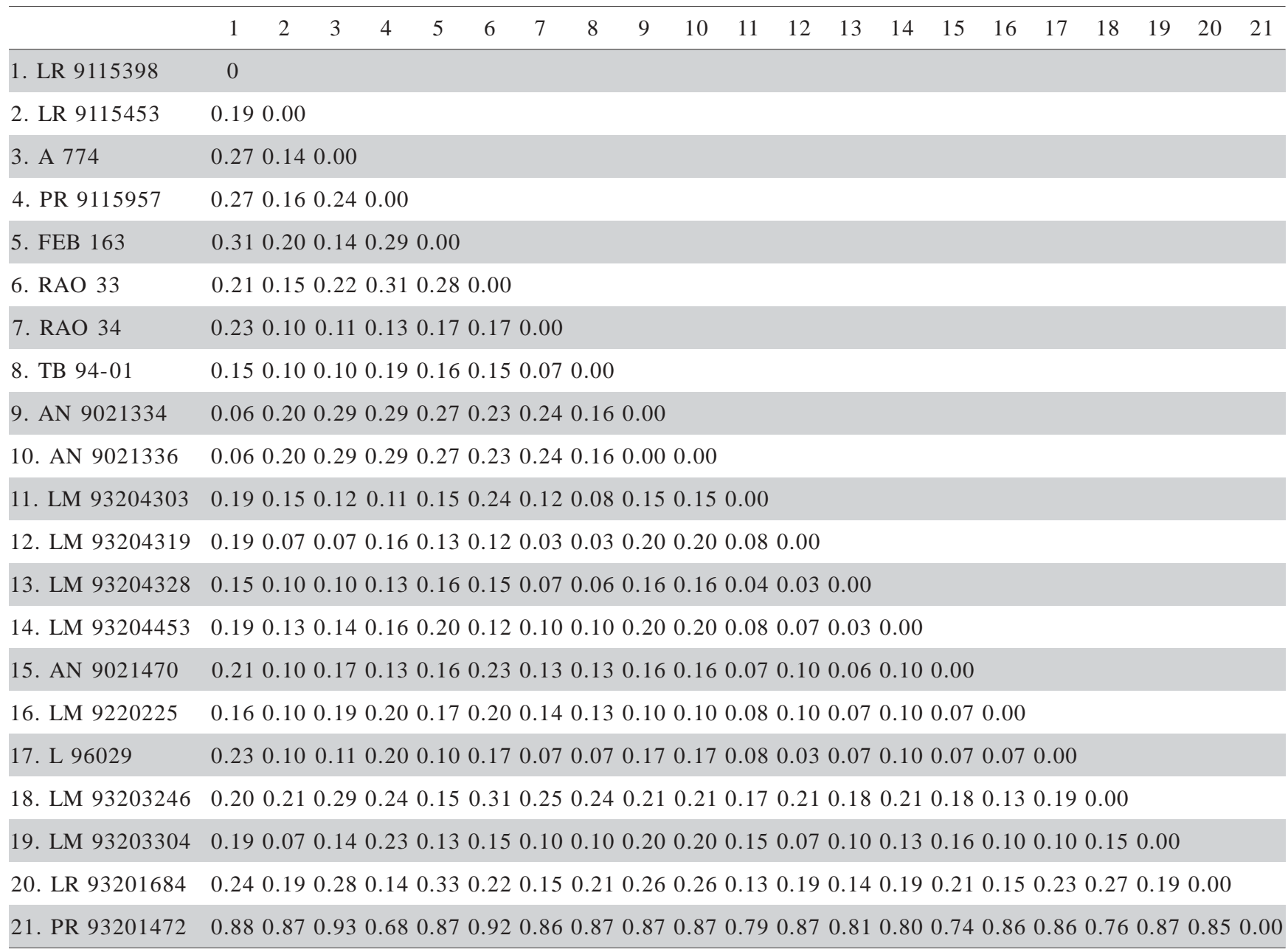

The second group was formed by 17 lines, with different seed colors, originated from 50 parents, with a genetic distance between 0.03 and 0.33 between them (Figure 1). The smallest distance was observed between the cream beige seed lines LM 93204319 (12) and LM 93204328 (13), which have identical genealogies. The highest distance was observed between lines FEB 163 (5) and LR 93201684 (20). Despite the fact that lines LR 93201684 (20) and RAO 33 (6) have Andean cultivar 'Pompadour' as progenitor in their pedigrees, they do not form a separate group. Probably those lines have low percentage of Andean genes in concordance with the small size of their seeds. Almost all lines of groups I and II were originated in Central America and Caribbean (Mexico, Costa Rica, Dominican Republic and Nicaragua), and all have small seeds, characteristic of the Mesoamerican gene pool (Tables 1, 2 and 3).

A third group, was formed only by manteigão line PR 93201472 (21) derived from a cross between the Andean cultivar 'Pompadour' originated in the Dominican Republic (Voysest, 1983) and cultivar 'Irai', of unknown origin. These lines, with medium size seeds pre-

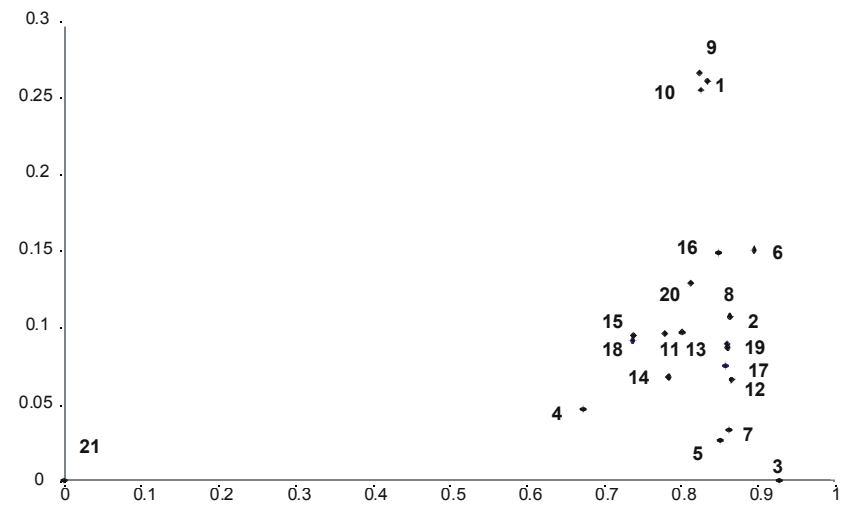

Figure 1- Graphic dispersion of the 21 common bean cultivars of the Bean Elite Regional Trials coordinated by Embrapa Rice and Beans.

sented the highest genetic distances in relation to the other lines tested (0.68 and 0.93) (Tables 1,2 and 3).

The low genetic variability of the parents used in the original crosses and the frequent use of the same parents for the development of these cultivars may have been the cause of low genetic variability observed in this work. 
For instance, the Mesoamerican cultivars/lines 'Jamapa', 'Carioca', 'Veranic 2' (selected from 'Jamapa'), 'Cornell 49-242', 'Tlalnepantla 64' (PI 207.262) and 'Tara' have been used for the development of most of the 17 lines in group II (Vasconcelos, 1995; Gepts et al., 1986; Koenig et al., 1990; Singh et al., 1991b; Voysest, 1983, 2000) (Tables 1 and 2). Voysest (2000) pointed out that Mesoamerican cultivars 'Jamapa' and 'Carioca', released in 1958 and 1966, respectively, are among the five cultivars most frequently grown in Latin America. Although these cultivars have been important because of their genetic stability and yield, their use as commercial cultivars and as parents in several breeding programs may have contributed for the reduction of the genetic variability of the new commercial cultivars in Brazil and other of Latin America countries. The cultivar/line 'Tara' and 'Tlalnepantla 64' (PI 207.262) are used as source of resistance to Xanthomonas axonopodis pv. phaseoli. The latter is also used as source of resistance for anthracnose. Cultivar 'Cornell 49-242' carries anthracnose and leaf spot resistance genes and is one of the 12 differential varieties used to classify pathotypes of the pathogens Colletotrichum lindemuthianum and Phaeoisariopsis griseola (Young \& Kelly, 1996; Pastor-Corrales, 1992; Pastor-Corrales \& Jara, 1995). Lines FEB 163, LM 93204303 and LM 93204453 have in common the same six gene donors described above. Lines TB 94-01, LM 9220225 and LM 93203304 have in their genealogies at least three of the above cultivars used as source of genes (Tables 1 and 2).

Although results show a low genetic variability among 17 bean lines (group II), derived from 50 cultivars (mostly Mesoamerican), there is no doubt that the Mesoamerican germplasm from different bean races (Mesoamerican, Durango and Jalisco) may contain important genetic variability to be incorporated into bean breeding programs. Indeed, Beebe et al. (2000) have demonstrated, by analyzing 269 Mesoamerican bean landraces, that this gene pool is an important source of genetic variability that remains to be explored.

Using 12 RAPD primers, the 21 lines could be classified into 3 groups, variation were detect in 17 lines of group II, duplicate lines were identified in groups I and II. This type of information is essential for germplasm conservation and improvement. Knowledge of the pedigree of a cultivar may be useful for the identification of sources of genes of interest and understand its role in genetic variability. However, the pedigree information is not always available for the breeder. In these cases, molecular markers can be used as an accurate tool to detect similarity/divergence and identificate duplicated bean lines or accessions among bean cultivars. Molecular analyses, in conjunction with morphological and agronomic evaluations of cultivars are recommended, because they provide complementary information and increase the resolving power of genetic diversity analysis (Singh, et al., 1991c).

\section{ACKNOWLEDGEMENTS}

Ana Lilia Alzate-Marin was supported by IICA. This work was supported by a grant from EMBRAPAArroz e Feijão and CNPq. Maria Regina Costa was the recipient of an undergraduate scholarship from FAPEMIG.

\section{REFERENCES}

ALVAREZ, M.T.; SÁENZ DE MIERA, L.E.; PEREZ DE LA VEJA, M. Genetic variation in common bean and runner bean of the Northern Meseta in Spain. Genetic Resources and Crop Evolution, v.45, p.243251,1998

ALZATE-MARIN, A.L.; COSTA, M.R.; BARROS, E.G.; MOREIRA, M.A. Characterization of the anthracnose resistance locus present in cultivar Ouro Negro (Honduras 35). Annual Report of the Bean Improvement Cooperative - BIC, v.44,p.115-116, 2001.

ARRAES, P.A.; BATISTA DE SOUZA, C.R. Tipos de faseolina em raças “crioulas de feijão no Brasil”. Pesquisa Agropecuária Brasileira, v.27, p.1219-1221, 1992.

BALARDIN, R.S.; PASTOR-CORRALES, M.A. Reação de germoplasma de Phaseolus vulgaris a nove raças de Colletotrichum lindemuthianum. Fitopatolologia Brasileira, v.15, p.269-273, 1990.

BASSETT, M.J. List of genes - Phaseolus vulgaris. Annual Report of the Bean Improvement Cooperative - BIC, v.39, p.3, 1996.

BEEBE, S.; SKROCH, P.W.; DUQUE, M.C.; PEDRAZA, F.; NIENHUIS, J. Structure of genetic diversity among Common Bean Landraces of Middle American origin based on correspondence analysis of RAPD. Crop Science, v.40, p.262-272, 2000.

CHASE, C.D.; ORTEGA, V.M.; VALLEJOS, C.E. DNA restriction fragment length polymorphisms correlate whit isozyme diversity in Phaseolus vulgaris L. Theoretical and Applied Genetics, v.81, p.806-811, 1991.

CIAT - Centro Internacional de Agricultura Tropical. Databases In Plant Genetics Resources. http://webpc.ciat.cgiar.org:8080/urg/ bean_search.jsp.(04-07-02).

CRUZ, C.D. Programa genes aplicativo computacional em genética e estatística. Viçosa: UFV, 1997. 390p.

CRUZ, C.D.; SORIANO, J.M. A methodology of genetic divergence analysis based on sample unit projection on two-dimensional space. Revista Brasileira de Genética, v.17, p.69-73, 1994.

DOYLE, J.J. ; DOYLE, J.L. Isolation of plant DNA from fresh tissue. Focus, v.12, p.13-15, 1990.

FALEIRO, F.G; VINHADELLI, W.S.; RAGAGNIN, V.A.; CORRÊA, R.X.; MOREIRA, M.A.; BARROS, E.G. RAPD markers linked to a block of genes confering rust resistance to the commom bean. Genetics and Molecular Biology, v.23,p.399-402, 2000.

FAO. Production Yearbook. Roma, v.52, p.94-95, 1998.

GEPTS, P. Phaseolin as an evolutionary marker. In: GEPTS, P. (Ed.). Genetic resources of Phaseolus beans, 1988. p.215-241.

GEPTS, P.; OSBORN, T.C.; RASHKA, K.; BLISS, F.A. Phaseolin-protein variability in wild forms and landraces of the common bean (Phaseolus vulgaris): evidence for multiple centers of domestication. Economic Botanic, v.40, p.451-468, 1986.

JOHNSON, W.C.; GEPTS, P. Influence of epistasis and diverse evolution on wide crosses of common bean. In: THE INTERNATIONAL CONFERENCE ON THE STATUS OF PLANT \& ANIMAL GENOME RESEARCH, San Diego, 1998. Summary W 83.

KOENIG, R.; SINGH, S.P.; GEPTS, P. Novel phaseolin types in wild and cultivated common bean (Phaseolus vulgaris, Fabaceae). Economic Botanic, v.44, p.50-60, 1990.

MAYEK-PEREZ, N.; LÓPEZ-CASTAÑEDA, C.; LÓPEZ-SALINAS, E.; CUMPIÁN-GUTIERREZ, J.; JOAQUIN-TORRES, I.C.; ACOSTAGALLEGOS, J.A. Resistance to Macrophomina phaseolina in common bean germplasm in Mexico. Annual Report of the Bean Improvement Cooperative - BIC, v.42, p.67, 1999. 
MÉTAIS, I.; AUBRY, C.; HAMON, B.; JALOUZOT, R.; PELTIER, D. Description and analysis of genetic diversity between commercial bean lines (Phaseolus vulgaris L.). Theoretical and Applied Genetics, v.101, p.1207-1214, 2000

NIENHUIS, J.; SKROCH, P.; BEEBE, S.; THOME, J.; PEDRAZA, F. Comparison of genetic diversity between random samples from core and reserve collections of Phaseolus vulgaris L. Annual Report of the Bean Improvement Cooperative - BIC, v.39, p.112-113, 1996.

PASTOR-CORRALES, M.A. Recomendaciones y acuerdos del primer taller de antracnosis en América Latina. In: PASTOR-CORRALES, M. (Ed.) La antracnosis del frijol común, Phaseolus vulgaris, en América Latina.. Cali: CIAT, 1992. p.240-250. (Doc. de trabajo $n^{\circ}$. 113).

PASTOR-CORRALES, M.A.; JARA, C.E. La evolución de Phaeoisariopsis griseola con el fríjol común en América Latina. Fitopatologia Colombiana, v.19, p.15-22, 1995.

Phaseolus Crop Germplasm Committee Report. http://www.ars-grin.gov/ npgs/cgc reports/phascgc.htm. (1996)/(02-07-02).

SANDLIN, C.M.; STEADMAN, J.R.; ARAYA, C.M. Isolates of Uromyces appendiculatus with specific virulence to landraces of Phaseolus vulgaris of Andean origin. Plant Disease, v.83, p.108-113, 1999.

SINGH, S.; GUTIERREZ, A.; URREA, C.; KOENING, R.; NODARI, R.; GEPTS, P. Marker-based analysis of genetic diversity in common bean. Annual Report of the Bean Improvement Cooperative - BIC, v.33, p.15-16, 1990.

SINGH, S.P.; GEPTS, P.; DEBOUCK, D.G. Races of common bean (Phaseolus vulgaris, Fabaceae). Economic Botanic, v.45, p.379-396, 1991a.

SINGH, S.P.; NODARI, R.; GEPTS, P. Genetic diversity in cultivated common bean I. Allozymes. Crop Science, v.31, p.19-23, 1991 b.

SINGH S.P.; GUTIERREZ, J.A.; MOLINA, A.; URREA, C.; GEPTS, P. Genetic diversity in cultivated common bean II. Marker-based analyses of morphological and Agronomic traits. Crop Science, v.31, p.23-29, 1991c.
SONNANTE, G.; STOCKTON, T.; NODARI, R.O.; BECERRA VELÁSQUEZ, V.L.; GEPTS, P. Evolution of genetic diversity during the domestication of common-bean (Phaseolus vulgaris L.). Theoretical and Applied Genetics, v.89, p.629-635, 1994.

THOME, J.; BEEBE, S.; GONZALEZ, O.; DUQUE, M.C. AFLP analyses of the wild Phaseolus vulgaris core collection. Annual Report of the Bean Improvement Cooperative - BIC, v.39, p.176-177, 1996.

URREA, C.; MIKLAS, P.P.; BEAVER, J.; RILEY, R. A codominant randomly amplified polymorphic DNA (RAPD) marker useful for indirect selection of BMGV resistance in common bean. Journal of the American Society for Hor ticultural Science, v.121, p.1035-1039, 1996.

USDA. ARS. National Genetic Resources Program. Germplasm Resources Information Network - (GRIN). [Online Database] National Germplasm Resources Laboratory, Beltsville, Maryland. www.arsgrin.gov/cgi-bin/npgs/html/acc search.pl. (02-07-02).

VASCONCELOS, M.J.V. Avaliação da variabilidade genética de cultivares de feijão (Phaseolus vulgaris L.) pelo uso de marcadores moleculares RAPD. Viçosa: UFV, 1995. 54p. (Dissertação - M.S.)

VOYSEST, O. Variedades de frijol em América Latina y su origen. Cali: CIAT, 1983. 87p.

VOYSEST, O. Mejoramiento genético del frijol (Phaseolus vulgaris L.). Legado de variedades de América Latina 1930-1999. Cali: CIAT, 2000.195p

WILLIAMS, J.; KUBELIK, A.; LIVAK, K.; RAFALSKI, A.; TINGEY, S. DNA polymorphisms amplified by arbitrary primers are useful as genetic markers. Nucleic Acids Research, v.18, p.6531-6535, 1990.

YOUNG, R.A.; KELLY, J.D. RAPD markers flanking the Are gene for anthracnose resistance in common bean. Journal of the American Society for Horticultural Science, v.121, p.37-41, 1996.

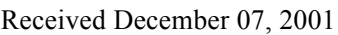

